\title{
Continuous-wave, pair-pumped laser
}

\author{
Ping Xie and Stephen C. Rand \\ Department of Electrical Engineering and Computer Science, University of Michigan, 1301 Beal Avenue, Ann Arbor, Michigan 48109-2122
}

Received March 2, 1990; accepted May 8, 1990

We report room-temperature operation of what we believe is the first continuous-wave laser that relies exclusively on cooperative upconversion by coupled ion pairs to achieve population inversion.

Conventional excitation of laser media raises individual atoms or ions directly to energy levels above the emitting level. ${ }^{1}$ However, as we show here, energy states lying well above those populated by incident light can be reached by spontaneous, cooperative upconversion processes at rates sufficient to sustain stable, steady-state population inversions. Here we demonstrate $\mathrm{cw}$ laser action from upconverted states without significant contributions from multiphoton absorption. This novel operating principle holds promise as a means of pumping short-wavelength solid-state lasers without the need for radiation sources of still shorter wavelength, without the need for multiphoton absorption coincidences, and without the usual problems of color-center formation and short extinction length for pump light near the band edge of a solid.

While the cooperative upconversion pair process was previously found to account for modified kinetics in erbium lasers, ${ }^{2}$ it has not been shown to be capable on its own of sustaining continuous inversion, as we show here. A variety of pulsed ${ }^{3}$ and $\mathrm{cw}$ upconver$\operatorname{sion}^{4,5}$ lasers pumped by sequential multiphoton absorption processes at low temperatures have been reported. Also, earlier studies of upconversion lasers included those of pulsed operation of pair-pumped solid-state erbium lasers by Pollack et al. ${ }^{6}$ and Rand et $a .^{7}$ In addition, conventional excitation was used to achieve cw operation on the 2.8- $\mu \mathrm{m}$ transition of erbium in $\mathrm{CaF}_{2}$ (Ref. 6) and other hosts. ${ }^{8}$ Here, however, steady-state inversion of the upper laser level is achieved exclusively by upconversion due to spontaneous Er-Er interactions in the excited state. We also discuss why earlier studies ${ }^{2,6,7}$ or traditional laser analysis could not have predicted that true $\mathrm{cw}$ pairpumped laser operation is even possible.

The energy-level diagram of the $\mathrm{Er}^{3+}$ ion is shown in Fig. 1. The crosses on level ${ }^{4} I_{13 / 2}$ represent erbium ions initially prepared in this level by resonant absorption of light from a cw NaCl color-center laser operating at $1.51 \mu \mathrm{m}$. Pair interactions of near-neighbor ions can induce cooperative upconversion as shown in the figure, and similar processes have been the subject of research in gases since the work of Welch ${ }^{9}$ and in solids since the time of Dieke. ${ }^{10}$ The curved arrows indicate the dynamics schematically, with ion 2 losing energy by returning to the ground state and ion 1 gaining energy by upconversion to level ${ }^{4} I_{9 / 2}$, which then relaxes rapidly [lifetime $\sim 7 \mu$ sec (Ref. 11)] to populate the upper laser level ${ }^{4} I_{11 / 2}$. In dilute $\mathrm{Er}: \mathrm{CaF}_{2}$ crystals the transition ${ }^{4} I_{11 / 2}-{ }^{4} I_{13 / 2}$ is self-quenched because the ${ }^{4} I_{13 / 2}$ lifetime $(28 \mathrm{msec})$ exceeds that of ${ }^{4} I_{11 / 2}$ (13 msec) by roughly a factor of 2 at low excitation rates. ${ }^{6}$ However, Er-Er interactions in concentrated crystals make the ${ }^{4} I_{13 / 2}$ lifetime excitation dependent. As a result, even when the lower laser level is pumped directly, as in the study reported here, the lower-state lifetime decreases with increasing occupation, and it becomes possible to achieve steady-state inversion from upconverted states without the assistance of multiphoton excitation.

This surprising result is in agreement with an exact analysis ${ }^{12}$ in which the comparative rates of two-photon and two-atom pumping of the high-lying ${ }^{4} I_{9 / 2}$ state may be varied. In our model each manifold is represented as a single level to furnish a simple but realistic picture of the basic dynamics. Within the rate-equation approximation, diagonal density matrix elements for the nonlinear four-level system are

$$
\begin{aligned}
\frac{\mathrm{d}}{\mathrm{d} t} \rho_{11}= & \gamma_{41} \rho_{44}+\gamma_{31} \rho_{33}+\lambda_{21} \rho_{22}+\alpha \rho_{22}{ }^{2}-B_{12} I\left(\rho_{11}-\rho_{22}\right), \\
\frac{\mathrm{d}}{\mathrm{d} t} \rho_{22}= & \gamma_{42} \rho_{44}+\gamma_{32} \rho_{33}-\gamma_{21} \rho_{22}-\kappa_{23}\left(\rho_{22}-\rho_{33}\right) \\
& -2 \alpha \rho_{22}{ }^{2}+B_{12} I\left(\rho_{11}-\rho_{22}\right) \\
\frac{\mathrm{d}}{\mathrm{d} t} \rho_{33}= & \gamma_{43} \rho_{44}-\gamma_{3} \rho_{33}-\kappa_{23}\left(\rho_{33}-\rho_{22}\right) \\
\frac{\mathrm{d}}{\mathrm{d} t} \rho_{44}= & \alpha \rho_{22}{ }^{2}-\gamma_{4} \rho_{44},
\end{aligned}
$$

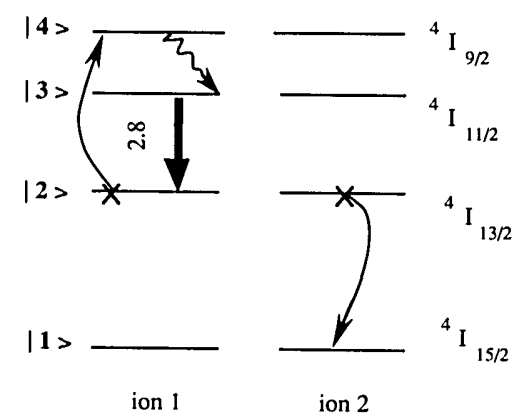

Fig. 1. Two-atom cooperative upconversion process responsible for steady-state inversion of level ${ }^{4} I_{11 / 2}$ with respect to level ${ }^{4} I_{13 / 2}$ in Er:CaF ${ }_{2}$. The initial excited state, with both $\mathrm{Er}$ ions in the ${ }^{4} I_{13 / 2}$ state, is prepared by cw irradiation at $1.51 \mu \mathrm{m}$ on the ${ }^{4} I_{15 / 2}{ }^{4} I_{13 / 2}$ absorption resonance. 
where $\gamma_{i j}$ is the spontaneous relaxation rate from level $i$ to $j, B_{i j}$ is the induced absorption rate, $\alpha$ is the pair upconversion coefficient, $\kappa_{i j}$ is the rate of stimulated emission, and $\gamma_{4}=\gamma_{43}+\gamma_{42}+\gamma_{41}, \gamma_{3}=\gamma_{32}+\gamma_{31}$ are total decay rates of levels 4 and 3 .

In Eq. (2) the term $-2 \alpha_{22}{ }^{2}$ accounts for the loss of two ions simultaneously from level 2 during the cooperative upconversion process. One of these ions is destined for level 4 and one for level 1, giving rise to the positive $\alpha$ terms in Eqs. (1) and (4). This process populates levels higher than level 2. Steady-state solutions for the populations when two-photon absorption is turned off $\left(B_{24}=0\right)$ furnish the condition for population inversion between levels 2 and $3\left(\rho_{33} / \rho_{22}>1\right)$ :

$$
\alpha>\alpha_{\mathrm{th}}\left[1+\frac{\gamma_{43}\left(\gamma_{21}+\gamma_{31}\right)+\gamma_{3}\left(\gamma_{4}+\gamma_{41}\right)}{I B_{12}\left(3 \gamma_{43}+\gamma_{3}\right)}\right] \text {. }
$$

Here $\alpha_{\text {th }}=\gamma_{3} \gamma_{4}\left(3 \gamma_{43}+\gamma_{3}\right) / N \gamma_{43}{ }^{2}$ and $N=N_{1}+N_{2}+$ $N_{3}+N_{4}$. Inequality (5) shows that (ignoring cavity losses) steady-state inversion is possible for any nonzero pump intensity $I$, provided that the upconversion coefficient is sufficiently large.

For coupled-atom systems, however, the theoretical requirement for inversion given in inequality (5) does not guarantee stable $\mathrm{cw}$ operation in the same way as for conventional lasers. It is well known that, in oscillator systems with strong nonlinear coupling, ${ }^{13}$ unstable and even chaotic steady states are frequently encountered. Hence it is necessary to introduce small perturbations, linearize system equations about their steady-state values, and consider coherences to investigate stability of $\mathrm{cw}$ cooperatively pumped lasers.

We have performed such an analysis, ${ }^{12}$ using the Routh-Hurwitz criterion for stability. ${ }^{14}$ The steadystate inversion due to Er pair interactions is predicted to be stable if $\gamma_{43}$ is large compared with other decay rates in the system. According to our calculations, true cw operation (without chaotic behavior or sustained oscillations) should be achievable whenever the final state in the cooperative transition relaxes quickly, effectively preventing feedback through the reverse transition (cross relaxation). This result is relevant to our coupled-atom system in $\mathrm{Er}: \mathrm{CaF}_{2}$, where the ${ }^{4} I_{9 / 2}$ lifetime is indeed short. In the opposite, coherent coupling limit, however, transient or sustained oscillations and population pulsations are expected to be prevalent in pair-pumped processes.

For the experiment, a crystal of $5 \% \mathrm{Er}: \mathrm{CaF}_{2}$ was used as the pair-pumped laser medium. It consisted of a 3-mm-thick disk prepared with one flat surface and one convex surface of radius $2.5 \mathrm{~cm}$. Both surfaces were antireflection coated in the range 1.4-1.6 $\mu \mathrm{m}$, and between 2.7 and $2.9 \mu \mathrm{m}$ the curved surface gave total reflection and the flat served as a $2 \%$ output coupler. The sample was pumped longitudinally at room temperature with a $\mathrm{cw} \mathrm{NaCl}$ color-center laser focused by a $5-\mathrm{cm}$ lens, absorbing $74 \%$ of radiation at $1.51 \mu \mathrm{m}$. For incident intensities above a threshold of $105 \mathrm{~mW}, \mathrm{TEM}_{00}$ laser emission was observed at 2.75 $\mu \mathrm{m}$, as shown in Fig. 2. Cw output of $9 \mathrm{~mW}$ was obtained with $200-\mathrm{mW}$ absorbed pump power, for an overall efficiency of $4.5 \%$. Laser output was monitored through a notch filter at $2.8 \mu \mathrm{m}$ (FWHM $10 \mathrm{~nm}$ ), using a fast InAs photodiode (rise time $\sim 5$ nsec) and a thermopile. Amplitude fluctuations at twice threshold were $-15 \mathrm{~dB}$ below the dc level within the measurement bandwidth (dc $1 \mathrm{MHz}$ ).

Before the onset of saturation, the output power of the laser increased quadratically with incident power in excess of threshold. This behavior established the pumping mechanism as either two-photon absorption or pair-mediated upconversion. (Reabsorption of resonance fluorescence cannot produce population inversion on a self-terminating transition.) We distinguished between these two possibilities with the timeresolved fluorescence measurements described next.

The time dependence of the ${ }^{4} I_{11 / 2}$ population was monitored directly by detecting fluorescence emission at $985 \mathrm{~nm}$ with a fast photomultiplier following shortpulse excitation of the laser medium. A rectangular pulse of $30-\mu \mathrm{sec}$ duration and $100-\mathrm{mW}$ peak power was selected acousto-optically from the cw pump beam for this purpose. Results are shown in Fig. 3(a).

The absorption of two pump photons must necessarily occur within the incident pulse duration. A calculation in which two-photon absorption accounts for $1 \%, 5 \%$, or $40 \%$ of the upper-state population at short times results in the curves of Fig. 3(b), indicating that observed fluorescence would exhibit an obvious step by the end of the pulse in these cases. The measured rise of fluorescence, though, reveals different behavior. Instead, it shows virtually no prompt emission and appears over an interval consistent with the lifetime of the ${ }^{4} I_{13 / 2}$ state in which pair interactions occur. These data immediately rule out contributions from two-photon absorption greater than approximately $1 \%$, making it clear that long before the rate of two-atom cooperative upconversion reaches its peak, it is already 100 times more effective than two-photon absorption at the power levels of this experiment. A detailed fit of the temporal evolution in Fig. 3(a), followed by extrapolation to steady-state populations, reveals that two-photon excitation rate actually accounts for much less than $1 \%$ of the steady-state inversion. ${ }^{12}$

At present only a rough comparison of observed threshold intensity with that predicted from inequality (5) is possible. Using the values for decay rates given in Refs. 6 and 11, we find that $\alpha_{t}=2.5 \times 10^{-19}$ $\mathrm{cm}^{3} \mathrm{sec}^{-1}$. Together with the only available estimate

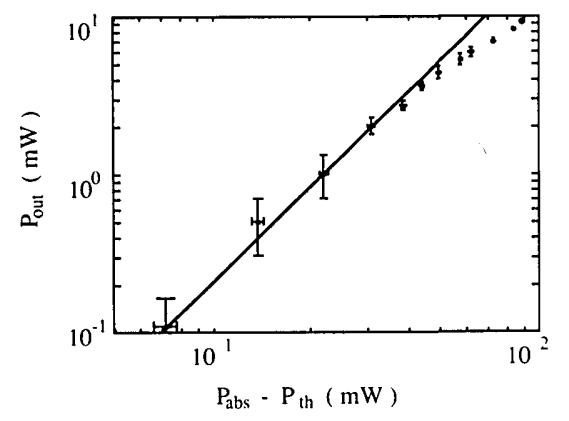

Fig. 2. Log-log plot of cw pair-pumped erbium laser output versus absorbed incident power above threshold. The solid curve has a slope of 2 , illustrating the quadratic dependence followed at low powers. The onset of saturation is evident at high power. 


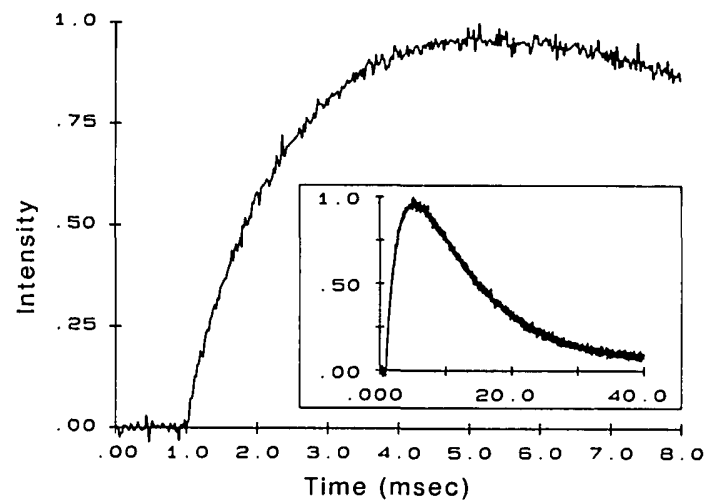

(a)

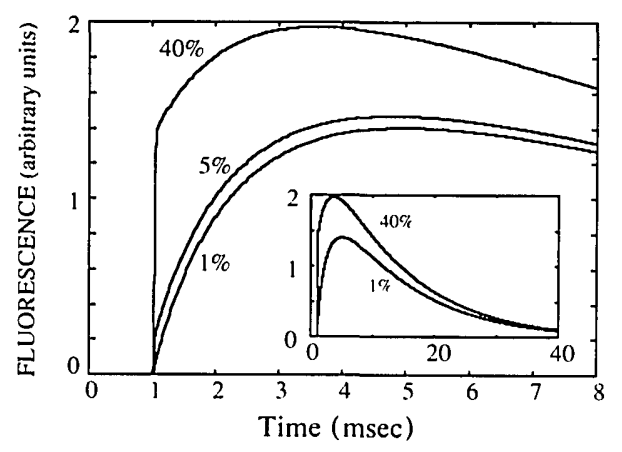

(b)

Fig. 3. (a) Fluorescence intensity at $985 \mathrm{~nm}$ versus time due to sample excitation at $t=1 \mathrm{msec}$ by an acousto-optically tailored pulse of $30-\mu \mathrm{sec}$ duration. Peak intensity of the pulse $(100 \mathrm{~mW})$ equals the average power level used in $\mathrm{cw}$ excitation of the air-pumped laser. (b) Calculation of expected evolution of fluorescence when various proportions of excited-state population $\rho_{33}$ are furnished by two-photon absorption during the $30-\mu \mathrm{sec}$ pulse interval.

of the upconversion coefficient, $\alpha=0.32 \times 10^{-16} \mathrm{~cm}^{3}$ $\mathrm{sec}^{-1}, 15$ this yields $I_{t}=2.1 \times 10^{4} \mathrm{~mW} / \mathrm{cm}^{2}$. Considering that our $\alpha_{t}$ calculation ignores output coupling, and that incident power is not uniform over the nonlinear gain region, this estimate compares rather favorably with the observed threshold of $3.2 \times 10^{5} \mathrm{~mW} /$ $\mathrm{cm}^{2}$ for an estimated spot radius of $100 \mu \mathrm{m}$. Internal losses due to water absorption in the $\mathrm{CaF}_{2}$, pair upconversion from ${ }^{4} I_{11 / 2}$, and higher-order upconversion processes have been ignored, and the true proportion of erbium impurities occurring in pair configurations is currently unknown. Contributions from these factors are expected to lead to increased estimates of $I_{t}$ and to improved agreement with experiment.

In summary, we have demonstrated a cw laser pumped exclusively by cooperative upconversion from pairs of coupled atoms. Its detailed implementation as a solid-state erbium laser is remarkable in that $\mathrm{cw}$ operation is achieved on a self-quenched transition with lower-laser-level pumping. Its unoptimized efficiency is high enough for us to suggest that other cooperative pumping schemes should be of interest for short-wavelength solid-state lasers. Indeed, we recently operated a cw Er upconversion laser at $0.855 \mu \mathrm{m}$ with $1.5-\mu \mathrm{m}$ cooperative upconversion pumping, again on a self-terminating transition with lower-laser-level pumping. This general approach therefore offers the prospect of new $\mathrm{cw}$, rare-earth lasers in other highly doped crystals on both self-quenched and unquenched transitions.

Note added in proof: The excitation mechanism of the pulsed, pair-absorption-pumped laser reported in $\mathrm{Ba} / \mathrm{Tl}$ vapor ${ }^{16}$ was quite distinct from that reported here. It relied on pair absorption on a collision-induced transition, a stimulated rather than a spontaneous process. Also, our result is fundamentally different from that of Quarles et al., ${ }^{17}$ in which spontaneous cross relaxation (cooperative downconversion) of Tm pairs achieved inversion of $\mathrm{Ho}$ after an additional energy-transfer step.

This research was sponsored by the U.S. Air Force Office of Scientific Research under contract F4962088-C-0079.

\section{References}

1. See, for example, A. E. Siegman, Lasers (University Science, Mill Valley, Calif., 1986).

2. E. V. Zharikov, N. N. Il'ichev, S. P. Kalitin, V. V. Laptev, A. A. Mulyutin, V. V. Osiko, P. O. Pashinin, A. M. Prokhorov, Z. S. Saidov, V. A. Smirnov, A. F. Umyskov, and I. A. Scherbakov, Kvant. Elektron. (Moscow) 13, 973 (1986).

3. L. F. Johnson and H. J. Guggenheim, Appl. Phys. Lett. 19, 44 (1971).

4. A. J. Silversmith, W. Lenth, and R. M. Macfarlane, Appl. Phys. Lett. 51, 1977 (1987); R. M. Macfarlane, F. Tong, A. J. Silversmith, and W. Lenth, Appl. Phys. Lett. 16, 1300 (1988).

5. R. A. McFarlane, Appl. Phys. Lett. 54, 2301 (1989).

6. S. A. Pollack, D. B. Chang, and N. L. Moise, J. Appl. Phys. 60, 4077 (1986).

7. S. C. Rand and S. A. Pollack, in Digest of OSA Annual Meeting (Optical Society of America, Washington, D.C., 1987), paper MD5, p. 21.

8. G. J. Kintz, R. Allen, and L. Esterowitz, Appl. Phys. Lett. 50, 1553 (1987).

9. H. L. Welsh, M. F. Crawford, J. C. F. MacDonald, and O. A. Chisholm, Phys. Rev. 83, 1264 (1951).

10. F. Varsanyi and G. H. Dieke, Phys. Rev. Lett. 7, 442 (1961); for a review of cooperative radiation processes in solids, see S. Hufner, Optical Spectra of Transparent Rare Earth Compounds (Academic, New York, 1978), Chap. 5 .

11. N. P. Barnes, R. E. Allen, L. Esterowitz, E. P. Chicklis, M. G. Knights, and H. P. Jenssen, IEEE J. Quantum Electron. QE-22, 337 (1986).

12. P. Xie and S. C. Rand, "Nonlinear dynamics of pairpumped processes," to be submitted to Phys. Rev. B. An earlier solution of this problem ${ }^{6}$ addressed only a special case and did not include a stability analysis, which is essential for nonlinear dynamic systems.

13. H. G. Winful and S. S. Wang, Appl. Phys. Lett. 53, 1894 (1988).

14. See, for example, I. N. Bronshtein and K. A. Semendyayev, Handbook of Mathematics (Van Nostrand Reinhold, New York, 1985), p. 419.

15. H. Chou, MIT Technical Rep. 26 (Massachusetts Institute of Technology, Cambridge, Mass., 1989), p. 62.

16. R. W. Falcone and G. A. Zdasiuk, Opt. Lett. 5, 155 (1980).

17. G. J. Quarles, A. Rosenbaum, C. L. Marquardt, and L. Esterowitz, Appl. Phys. Lett. 55, 1062 (1989). 